\title{
Study on the Origin of Nantong Blue Calico
}

\author{
Bing-ying $\mathrm{OU}^{1, \mathrm{a}}$, Hong-guang $\mathrm{YE}^{1, \mathrm{~b}}$ and Bin $\mathrm{LI}^{1, \mathrm{c},{ }^{*}}$ \\ ${ }^{1}$ College of Fashion, Wuhan Textile University, Wuhan, 430073, China \\ a995901055@qq.com, b36001463@qq.com, clibin790121@qq.com
}

\section{Keywords: Blue calico, Origin, Nantong.}

\begin{abstract}
The study on the origin of Nantong blue calico can start from two aspects, i.e. the origin of dye and the origin of dyeing technique. From the dye aspect, Nantong began to plant indigo plant (polygonum tinctorium) in late Ming Dynasty and early Qing Dynasty, which provided the raw materials for the development of blue calico. From the dyeing technique aspect, Nantong blue calico was developed on the basis of "Yaoban cloth". The appearance and development of dyeing technique of Nantong blue calico is closely linked with the development of dyeing technique in Song Dynasty, the death of Jiaxie technique and clothing color system.
\end{abstract}

\section{Introduction}

China's textile history generally believes that Nantong blue calico originates from the "Yaoban cloth" of Jiading town and Anting town (now belong to Shanghai) in the Song Dynasty, and it was created by a man whose surname was Gui. However, through in-depth study, the author believes that the appearance of Nantong blue calico has its historical inevitability and fortuity. As the predecessor of Nantong blue calico, "Yaoban cloth" it was the product of Chinese traditional dyeing art developed to a certain degree. If there were no traditional Chinese anti-dye techniques, especially the development of Jiaxie and Laxie techniques, it is not possible to generate "Yaoban cloth". At the same time, the folk Jiaxie technique was prohibited by the authority in the Song Dynasty, which had undoubtedly triggered the birth and popularity of "Yaoban cloth". So this is a kind of historical fortuity. Similarly, the appearance and development of Nantong blue calico was inevitably link with the excellent geographical environment, stable social order and the cultivation of polygonum tinctorium which was introduced by Cantonese mercers from Guangdong to Nantong in late Ming and early Qing Dynasty.

\section{Discussions and Analyses}

\section{Analysis of the Origin of Dye for Nantong Blue Calico}

The generation of dyeing technique of Nantong blue calico is based on planting blue dye plants. As early as three thousand years ago, Chinese had mastered the dyeing technology by plant material. As recorded in Zhouli Diguan, person who is in charge of dyeing cloth and silk "take charge of the plants which can be used for dyeing in spring and autumn and store them after weighing them and wait to hand out if need be used." It is obvious that there has been special person who is responsible for dye collection and dyeing dyed fabrics. As for the color of the dye, according to the book Xiaxiaozheng which is finished in the spring and autumn period (770 BC 476 BC), "in May, begin to plant indigo plant", so at the time, people have begun to plant the plant dyes used for indigo print-indigo plant (polygonum tinctorium). Before the spring and autumn period, indigo plant was used for dyeing only at season of harvesting indigo plant. Through cutting up and steeping the indigo plant, color liquid 
is produced. The color liquid dye should be used for dyeing as soon as possible, otherwise there will be a precipitation in color liquid so that it cannot be used for dyeing. Therefore, the harvest season of indigo plant is the time to dye fabric, and dye maker are very busy, as the description in Classic of Poetry-Xiaoya, "collecting the indigo plant all day, cannot filling the pocket". From the spring and autumn period to the warring states period (475 BC 221 BC), after exploring of dyer for long period, they finally found the craft making indigo plant into indigo blue and reverting indigo blue to blue dye liquor, so that it can be dyed blue in all seasons. As said in QuanXuepian written by Xunzi (about 313 BC 238 BC), "the dye extracted from the indigo is bluer than the plant". In this, "green" refers to the "indigo blue" and "blue" refers to "the indigo plant". It is proved that dyer in spring and autumn and warring states period have mastered the technology of producing indigo blue. The earliest specific record about the technology of extracting indigo from dye liquor of indigo plant in China is showed in Qimin Yaoshu-Zhonglan written by Jia Sixie in the Northern Wei dynasty (386 AD 557 AD), "in July make a hole, make it suffer hundreds of beams, make the wheat into mud and fill the hole with the mud, make it five inches deep to shadow walls, cut down indigo plant and place inversely it in the hole, pour into water, put wood and stone on it and make it wholly submerged. Hot it for one night, chill it for one night again, dry it, place it into urn, making ten stone urns, add lime of one dou and five liters, flip it by hand, stop a moment. Make it clear and purge water, make another small hole, store indigo plant in hole. Until is up to the time, get it out of urn, indigo blue is made." It is obvious that production technique of indigo blue has been quite mature in the Northern Wei Dynasty. Nantong had a tradition of planting indigo plant and producing indigo blue long ago. Nantong had the record of paying tribute indigo blue to royal or imperial court in Ming Dynasty Jia-jing period $(1522 \sim 1566)$, just at that time the raw materials for producing indigo blue was isatis tinctoria of which quality not as good as indigo plant.

Planting indigo plant in Nantong began in the late Ming and early Qing dynasties. Draper from Guangzhou brought seed of indigo plant. Then dyehouse and production process of dyeing begun to develop slowly in Nantong. According to the records of Nantong county annals, there are more than nineteen manual dyehouses registered in Dyeing Bureau. During the period of Emperor Guangxu, Tongzhou Zhi said that "plant indigo plant into the blue river, cut down in May as first indigo plant, cut down in July as second indigo plant, prepare a pool of water, pour water into lime, stir for thousands of times, take out the water, the indigo blue is produced. It is used for dyeing cloth, namely small green cylinder". Indeed indigo plant being widely planted in Nantong, developed production technology of indigo blue and appearance of a large number of dyes provide a good soil for the development of printing and dyeing technology of indigo print in Nantong.

\section{Analysis of the Origin of Dyeing Technique for Nantong Blue Calico}

The origin of dyeing technique for Nantong blue calico has direct and indirect aspects, including three sources:

\section{The Dyeing Techniques of Nantong Blue Calico come Directly from the "Yaoban Cloth" of Jiading and Anting Towns in Song Dynasty}

According to the Book Integration of Ancient and Modern Times-Zhifangdian, it says "Yaoban cloth" originated from Jiading and Anting town in Jiading period (1208 1224), Song Dynasty and it was created by a man whose surname was Gui. 
The cloths were wiped with plaster for dyeing, then dry it and remove the plaster, and then it was chequered with black and white. There were people, things, flowers, birds, poetries on it with different colors, and it was used for quilt and curtain." Jiading and Anting belong to Shanghai now, and Shanghai is just located across the river with Nantong. It can be concluded that the dyeing technique of Nantong blue calico was introduced in by the ancient Shanghai immigrants.

\section{The Dyeing Techniques of Nantong Blue Calico come Directly from Laxie Technique}

From the anti-dye principle of Nantong blue calico, it seems to absorb the style of Laxie technique, especially the Jiaxie wax-irrigating method of Lingnan Yao nationality. Zhouqufei (1135 1189), a man in Song Dynasty, described the bule calico of Yao people by plate batik method in book Lingwaidaida, which was finished in the fifth year of Chunxi (1178), while the predecessor of Nantong blue calico "Yaoban cloth" was produced in Jiading, Song Dynasty (1208 1224). The two events happened at least 30 years away from each other. Besides, the plate Laxie blue calico used by Yao people must had existed before Zhouqufei saw it. Therefore, I concluded that "Yaoban cloth" was most likely to absorb and improve the the anti-dye principle of plate Laxie technique of Yao people.

The Production of Pattern Plate for Nantong Blue Calico Indirectly Absorbs the Production Technique for Jiaxie Plate

The pattern plates of Nantong blue calico and Jiaxie both use engraved way for production. The difference is that the pattern is in the hollow area of pattern plate for Nantong blue calico, while the hollow area is the ground color for Jiaxie. At the same time, Nantong blue calico uses paper plate, while Jiaxie uses wood plate. Jiaxie was very popular in the folk dye houses in Tang Dynasty. Jiaxie was prohibited in folk in the Song Dynasty and then the "Yaoban cloth" emerged. The techniques always have a certain degree of inheritance. The carved pattern palte process used in the Jiaxie will affect the production of pattern plate of "Yaoban cloth". Therefore, the "Yaoban cloth" inherited the Laxie anti-dyeing process, while it inherited the carving process of Jiaxie pattern plate. Nantong blue calico was developed from the basis of "Yaoban cloth".

\section{Reasons of the Appearance of Dyeing Technique for Nantong Blue Calico}

The key of dyeing technique for Nantong blue calico is paper scraping technique, and the emergence of the anti-dyeing technology has some historical reasons:

The Use of Jiaxie Dyeing was Prohibited in Folk for Several Times in the Song Dynasty

History of the Song Dynasty-Yufuzhi records the imperial edict of the second year of Zhenghe (1112) "Houyuan made Xie clothe. Since the beginning of Yuanfeng, it was set as the March number, and also made for the guards clothing to identify treachery. Therefore, it was prohibited in folk dye house. Kaifeng House declared its ban regulations, and traders were not allowed to sell it." By this edict, it can be seen, the Song Dynasty had repeatedly prohibited people to produce Jiaxie pattern plate, and even those who were trading Jiaxie plate should be punished for a crime. In the government's ban, the folk Jiaxie technique must had withstood a great blow, and gradually declined and got lost in the end. 


\section{The Emergence of "Yaoban Cloth" Promotes the Development of China's Folk Blue Calico in Dyeing Industry}

The dyeing method of "Yaoban cloth" has three characteristics. Firstly, carved pattern plate took place of Jiaxie pattern plate. It was much easier to engrave carved paper plate than carved woord plate, which made it easy to conduct pattern plate production process. Secondly, bying using scraping anti-dyeing method, the success rate of dyeing became much higher than the Jiaxie dyeing. According to Xue Xunlang, an inheritor of Jiaxie dyeing technique in Wenzhou, he broke more than 500 pieces of cotton clothe in total in learning Jiaxie dyeing technique, the loss of nearly more than 300,000 RMB. So we can see Jiaxie anti-dyeing technique is quite demanding. However, the paper scraping anti-dyeing process used in the Nantong blue calico is much simpler. Only with a good paste, scratch it in the gap of the carved pattern plate and anti-dyeing pattern will be formed, which does not need to consider the clamping force in Jiaxie technique. Therefore, the paper scraping anti-dyeing technique is more efficient than the Jiaxie anti-dyeing technique.

\section{Blue becomes a Symbol of the Civilian Class in China's Ancient Costume System Due To the Color Restrictions}

The color of ancient Chinese costumes has a close relationship with identity levels. Each feudal dynasty advocated different colors of clothing. Xia Dynasty favored black, Shang Dynasty favored white, Zhou Dynasty favored red, Qin Dynsaty favored black, Han Dynasty favored red and Tang Dynasty favored yellow. However, each feudal dynasty generally follows the rule of "like orthodox color and unlike mixed color". In ancient China, the so-called "orthodox color" refers to cyan, red, yellow, white and black while "mixed color" refers to green, secondary red, blue, purple and black yellow. Therefore, in ancient times the blue color was a low-down color and used for the clothe color for the folk people. And any act that went beyond the clothe color system was banned. This is the reason why blue calico can keep popular in the folk.

\section{Conclusions}

Through the above analysis, we believe that firstly, the extensive cultivation of indigo plant in late Ming and Qing Dynasties in Nantong, the improvement of production technique for indigo blue and the emergence of a large number of dye houses provided a good soil for the development of Nantong blue calico technique; that secondly, the dyeing techniques of Nantong blue calico come directly from the "Yaoban cloth", indirectly from Laxie technique and the production of pattern plate absorbed the production technique of Jiaxie pattern plate. At the same time, the key of dyeing technique for Nantong blue calico is paper scraping technique, and the emergence of the anti-dyeing technology has some historical reasons. Firstly, the use of Jiaxie dyeing was prohibited in folk for several times in the Song Dynasty, forcing people to look for the alternative for Jiaxie dyeing process; secondly, the emergence of "Yaoban cloth" promotes the development of China's folk blue calico in dyeing industry; thirdly, the color restriction of China's ancient costume system made blue calico cater to this need. 


\section{References}

[1] SunYirang: ZhouLi ZhengYi (Beijing: Zhonghua Book Company, China1987).

[2] Liyong: Study of Nantong blue calico (Southeast Culture, China2005).

[3] Wang Ping: Chinese Activation of Blue Calico Exploration and Development (Journal of Zhejiang Wanli University, China2011).

[4] Chen Xianjin: Elegant Fu Blue and White Artful Dye Cotton Send Nostalgia, Nantong Blue Calico History and Characteristics (Hundred Schools in Arts, China2003).

[5] Wu Yuanxin: Jianghai bank will adopt blue -- Nantong blue calico process of inheritance and innovation (Nantong Shipping Vocational Institute of Technology, China2009).

[6] Sunlin: The costume colors and hierarchical relationships in ancient China (Modern Science, China2010). 